\title{
Contribution of somatosensory information to perception of the visual vertical with body tilt and rotating visual field
}

\author{
L. YARDLEY \\ University of Southampton, Southampton, England
}

\begin{abstract}
This study was designed to explore the role of somatosensory information from the trunk in the perception of the visual vertical. Twelve normal subjects and 1 subject with no somatosensory function below the neck attempted to set a line to the true vertical in the sitting and lying positions, first with a static visual background and then with rotation of the background about the line of sight. The absence of somatosensory information did not affect accuracy when the subjects were in the upright position. When lying horizontally, all control subjects experienced a substantial perceived tilt of the vertical in the direction of body tilt (the $A$ effect), but, in contrast, the subject lacking somatosensory function exhibited a small but consistent apparent tilt of the vertical in the opposite direction (the $E$ effect). This finding is discussed in relation to two competing hypotheses regarding the mechanisms subserving apparent displacement of the subjective vertical in tilted subjects.
\end{abstract}

Perception of the orientation of a vertical line is known to change as a function of the head or body angle of the observer. In general, a head tilt of up to $60^{\circ}$ induces a displacement of the apparent vertical in the opposite direction to head tilt (the $E$ effect), whereas tilts of greater magnitude provoke an increasing $A$ effect, whereby the apparent upright shifts in the same direction as the head and body tilt. These perceptual errors are assumed to be due to alterations, consequent upon head and body tilt, to the information relating to orientation provided by either the somatosensory system or the vestibular system. The net result is an apparent overcompensation for the displacement of the vertical relative to the head caused by small head tilts, and an undercompensation for larger tilts.

The evidence concerning the origin of the $A$ and $E$ effects is complex and inconclusive. The $E$ effect is in the direction consistent with a failure to take into account the automatic countertorsion of the eyes associated with head tilt. However, no consistent relationship has been found between the degree of ocular countertorsion and apparent displacement of the vertical (Merker \& Held, 1981), and the $E$ effect can be demonstrated in subjects without vestibular function (i.e., whose eyes do not tort). Indeed, although the combined $A$ and $E$ effects appear to parallel the function relating degree of body tilt to the shearing force acting on the utricles, remarkably similar patterns of illusion can be observed in normal subjects and in those with bilateral loss of vestibular function (Miller, Fregly, \& Graybiel, 1968). It has been suggested that the somatosensory system may play an increasing role in the

Correspondence may be addresed to Lucy Yardley, Department of Audiology, Institute for Sound and Vibration Research, University of Southampton, Southampton S09 5NH, England. determination of orientation with progressively larger body tilts (Schone, 1975). However, attempts to quantify this influence have been hampered by the immense difficulties involved in excluding or manipulating somatosensory information (Nyborg, 1971; Stoffregen \& Riccio, 1988).

Some form of intersensory integration of information from the visual, vestibular, and somatosensory systems is also assumed to subserve the illusory tilt of the vertical induced by continuous rotation of a large portion of the visual field about the line of sight. Dichgans, Diener, and Brandt (1974) established an interaction between the two illusions. When head tilt and background rotation were in the same direction, and would therefore normally induce an apparent tilt of the vertical in opposite directions, the visual field motion had the greatest effect on perceived orientation. However, head tilt and background rotation inducing a congruent direction of tilt generated an apparent tilt of the vertical that was greater than the added effects of these two conditions alone. This interaction was attributed to a reduction in the weight given to vestibular information when the head was tilted, owing to a reduction in otolithic sensitivity in this position. Nevertheless, changes in vestibular and somatosensory information resulting from head tilt were again inextricably confounded.

The aim of the present study was to clarify the role played by the vestibular and somatosensory systems in these illusions by comparing the performance of normal subjects with that of a subject who had lost virtually all somatosensory function below the neck. For this purpose, the subjects assessed the perceived orientation of the vertical in both the upright and horizontal positions, with and without rotation of the visual background. 


\section{METHOD}

\section{Subjects}

The experimental subject, N.L., had lost large myelinated sensory nerve function some 15 years previously as a result of a viral illness. He had no feeling in the trunk apart from very poorly localized sensations of deep pressure, pain, and fatigue; conventional nerve conduction studies revealed normal motor conduction but no sensory action potential (see Cole, Katifi, \& Sedgwick, 1986). The cranial nerves were unaffected, and normal audiovestibular and NVIII function was confirmed for the purpose of this study by caloric and auditory brainstem response testing. Immediately following his illness, N.L. was completely incapacitated, but over the next few years he gradually learned to control his movements without sensory feedback and is now able to live independently.

Six males and 6 females (age range 22-34 years) served as normal controls. None of the control subjects had any history of visual or audiovestibular impairment, or any previous experience with the orientation task.

\section{Apparatus}

A circular visual display, capable of rotating at a constant angular velocity in either direction, was generated by back-projecting the image of a clear Perspex disk ( $26 \mathrm{~cm}$ in diameter) onto a vertical neutral density Perspex screen $(122 \times 122 \mathrm{~cm})$ whose lower edge was $54 \mathrm{~cm}$ above floor level. Affixed to the center of the screen was an opaque circular plastic disk $13 \mathrm{~cm}$ in diameter. The clear (stimulus) disk was mounted horizontally over the projection surface of a $3 \mathrm{M}$ overhead projector situated $2.15 \mathrm{~m}$ directly behind the screen. The moving visual display was created by placing an acetate sheet, completely covered in a pattern of quasirandom dots of varying sizes, over the stimulus disk, which was maintained in position by three roller bearings and edge-driven by a variable-speed 12-V dc electric motor. A Leitz Pradovit 2-in. slide projector situated $2.4 \mathrm{~m}$ directly behind the center of the screen was fitted with a slide, which was opaque apart from a small slit. This was used to superimpose a projected white line $27 \mathrm{~cm}$ long and $0.5 \mathrm{~cm}$ wide onto the moving visual display. The line was centered on the rotational axis of the moving display, and its angle could be adjusted by manually rotating the lens barrel of the projector. The angle at which the line was set could be read off to an accuracy of $0.25^{\circ}$ from the position of a rod attached to the lens barrel relative to a clear semicircular protractor aligned precisely to the gravitational vertical and fixed to the main body of the projector.

The subject was seated in front of the screen with the head on an adjustable chinrest and the eyes level with the central disk. Peripheral vision was restricted to approximately $100^{\circ}$ of visual angle by lensless plastic goggles, and the distance from cornea to the disk at center screen was $43 \mathrm{~cm}$; thus, the subject's field of vision was entirely filled by the rotating visual display, apart from the opaque disk, which subtended $17.2^{\circ}$ of central vision.

\section{Procedure}

Using binocular vision, each subject was asked to fixate the disk at the center of the display. During "static" trials, the white line was then projected onscreen at a predetermined initial setting, and the subject indicated verbally whether the line should be moved left or right toward the vertical. The experimenter, who had no view of the position of the line during trials, proceeded to smoothly rotate the line in the required direction at a velocity of not more than $2 \% \mathrm{sec}$ until the subject reported that the line appeared to be verti$\mathrm{cal}$; the angle of the final line position was then read off from the protractor and recorded. The method of setting the orientation of the line by verbal directions was chosen to permit direct comparison with the settings made by N.L., who was, of course, unable to set the line by any manual method while watching the visual display. Although probably subject to greater measurement error than some alternative techniques, the results indicate that this method yields a high degree of accuracy when settings are averaged over several trials.

During "rotation" trials, the same procedure was followed, except that the background display commenced rotation, at a constant velocity of $36^{\circ} / \mathrm{sec}, 10 \mathrm{sec}$ before the initial line setting was projected onto the screen. As soon as the subject indicated that the line was vertical, it was removed from display and rotation of the background ceased. To minimize any possible adaptation effects, an intertrial interval of $60 \mathrm{sec}$ and alternating clockwise and counterclockwise rotations were employed. The number of initial line positions to the left or right of the vertical was counterbalanced, but the angles of these settings were randomly selected from between $0^{\circ}$ and $60^{\circ}$ from the vertical.

The subject made eight static line settings, followed by 16 rotation settings. After each trial, he/she was asked to report the strength of any illusion of self-motion experienced during the trial on a scale of 0-4 (very strong illusion of motion). The subject then completed an additional eight static trials and 16 rotation trials while lying horizontally on his/her left side on a couch with the head supported at $80^{\circ}$ from the vertical and the eyes again level with the disk at center-screen. This orientation was chosen in preference to a simple $90^{\circ}$ whole-body tilt because pilot investigations had indicated that subjects were more likely to attempt deliberate strategies for assessing the orientation of the vertical when they knew it was at right angles to the position of their heads.

\section{RESULTS}

In order to determine the validity of averaging rod settings made over several trials, the relationship between settings and trials was first examined. For this purpose, rod settings were expressed in terms of the signed deviation from the vertical in the clockwise direction. Table 1 shows the mean deviations from the vertical of the normal subjects as a function of trials. It can be seen that the effect of both body tilt and rotation of the visual field was quite consistent over trials, and showed no systematic habituation. To ensure that the group data did not obscure a pattern of adaptation over trials in a subset of subjects, Spearman's rank correlation between trial and rod setting was calculated for each subject separately; these proved universally nonsignificant. It was therefore considered appropriate to pool the data from the eight trials under each condition for subsequent analyses.

Interestingly, the strength of the reported sensation of rotation did diminish with repeated experience with the il-

Table 1

Mean Rod Settings of the 12 Normal Subjects in the Upright (Seated) and Horizontal (Lying) Positions with Static Clockwise (CW), and Counterclockwise (CCW) Background Rotation

\begin{tabular}{|c|c|c|c|c|c|c|}
\hline \multirow[b]{3}{*}{ Trials } & \multicolumn{6}{|c|}{ Position } \\
\hline & \multicolumn{3}{|c|}{ Upright } & \multicolumn{3}{|c|}{ Horizontal } \\
\hline & Static & $\mathrm{CW}$ & $\mathrm{CCW}$ & Static & $\mathrm{CW}$ & $\mathrm{CCW}$ \\
\hline $1-2$ & 0.09 & 8.01 & -9.83 & -19.40 & 4.86 & -35.24 \\
\hline $3-4$ & -0.09 & 7.86 & -9.80 & -21.02 & 2.43 & -35.36 \\
\hline $5-6$ & 0.20 & 8.21 & -8.86 & -20.13 & 0.29 & -36.80 \\
\hline $7-8$ & -0.19 & 7.43 & -8.00 & -19.80 & 0.97 & -33.89 \\
\hline
\end{tabular}

Note-Data are expressed in degrees of error from the gravitational vertical. A high degree of accuracy was achieved on the eight static (no visual field rotation) upright trials. The mean error induced by rotation trials and by the horizontal position is quite constant, showing no consistent or significant change over time. 


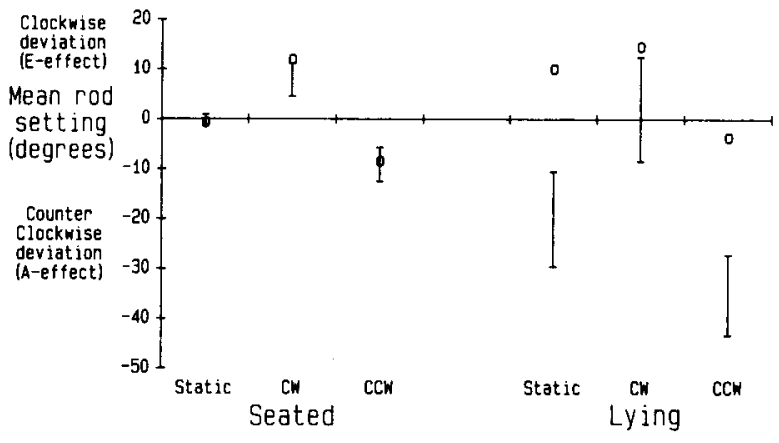

Figure 1. Ninety-nine percent confidence intervals for the mean deviation from the vertical of the rod settings of 12 control subjects under each condition: seated and lying, with static visual background and with clockwise $(\mathrm{CW})$ and counterclockwise $(\mathrm{CCW})$ rotation of the background. The settings of the experimental subject, N.L., are represented by open ovals. Negative settings indicate apparent displacement of the vertical in the counterclockwise direction.

lusion, from a mean value of 2.8 on the first two seated trials to only 1.6 on the last pair of seated trials. Withinsubject Spearman's rank correlations between ratings of the strength of the illusion of rotation and the error in setting the rod to the vertical were consequently weak and nonsignificant. No intersubject correlation was found between average subjective ratings of sensation of rotation and mean displacement of the apparent vertical (Spearman's rank correlations: 0.23 seated, -0.09 lying). The dissociation between reported sensations and rod settings was particularly evident in the case of N.L. Although he achieved approximately normal accuracy in judging the vertical in the seated position (see below), he reported an extremely strong reaction to the first trial with the rotating background and was able to save himself from falling only by tensing all his muscles and thereby consciously "freezing"' his position (his habitual strategy when threatened by a perturbation of posture). His ratings of motion sensation averaged a constant 3.3 during both the seated and the lying trials, compared to a control group average of 1.9 (maximum mean rating 2.9) under these two conditions.

The mean signed deviations from the vertical of the rod settings of the normal subjects over eight trials were then used to generate $99 \%$ confidence intervals for average set- tings under each condition; these are shown in Figure 1, with the precise means and standard deviations of both N.L. and one of the normal subjects given in Table 2. It can be seen from Figure 1 that the average settings of N.L. fell within the normal range while in the upright position, even with rotation of the visual background. In the horizontal position, with a static visual background, all of the normal subjects demonstrated a substantial A effect (tilt of the vertical in the same direction as their body tilt). In contrast, N.L. exhibited a clear E effect-a tilt of the apparent vertical in the opposite direction. This difference had a systematic and consistent effect on settings in the horizontal position with visual background rotation. During clockwise rotation, the conflict between the counterclockwise tilt induced by body position and the clockwise tilt provoked by the visual field motion resulted in settings from the control subjects centered roughly about the true vertical. In the case of N.L., the visual field motion and the $\mathrm{E}$ effect combined to produce settings slightly farther from the vertical, in the clockwise direction, than the normal subjects'. During counterclockwise background rotation, this pattern of interaction was reversed: in the normal subjects, the added effect of the two illusions led to an extreme apparent tilt of the vertical in the direction of their body tilt, whereas for N.L. the illusions approximately canceled out.

\section{DISCUSSION}

These results indicate that the absence of somatosensory information from below the neck has little effect on the magnitude of illusory displacement of the vertical produced by rotation of the visual background when the subject is seated upright. Previous authors (Dichgans et al., 1974; Young, Oman, \& Dichgans, 1975) have argued that vestibular, rather than somatosensory, information must be the most important factor limiting the extent of illusory tilt provoked by background rotation, since the perceived displacement of the vertical during visual field movement is enhanced by head tilts, which place the utricles in a position where their sensitivity is reduced. Certainly N.L. achieved essentially normal levels of accuracy in determining the vertical while upright, presumably relying primarily on information from the vestibular system,

Table 2

Mean ( $\pm S D$ ) Rod Settings of Subject N.L. and a Control Subject in the Upright (Seated) and Horizontal (Lying) Positions with Static, Clockwise (CW), and Counterclockwise (CCW) Background Rotation

\begin{tabular}{|c|c|c|c|c|c|c|c|c|c|c|c|c|}
\hline \multirow[b]{4}{*}{ Subject } & \multicolumn{12}{|c|}{ Position } \\
\hline & \multicolumn{6}{|c|}{ Upright } & \multicolumn{6}{|c|}{ Horizontal } \\
\hline & \multicolumn{2}{|c|}{ Static } & \multicolumn{2}{|c|}{$\mathrm{CW}$} & \multicolumn{2}{|c|}{$\mathrm{CCW}$} & \multicolumn{2}{|c|}{ Static } & \multicolumn{2}{|c|}{ CW } & \multicolumn{2}{|c|}{$\mathrm{CCW}$} \\
\hline & $M$ & $S D$ & $M$ & $S D$ & $M$ & $S D$ & $M$ & $S D$ & $M$ & $S D$ & $M$ & $S L$ \\
\hline N.L. & -1.0 & 1.4 & 11.7 & 2.1 & -8.7 & 2.1 & 9.8 & 3.2 & 14.3 & 7.3 & -3.9 & 4. \\
\hline Control & 0.3 & 2.5 & 5.9 & 1.9 & -12.0 & 2.4 & -23.0 & 4.9 & -6.0 & 6.0 & -42.5 & 3. \\
\hline
\end{tabular}

Note-Data are expressed as degrees of error from the gravitational vertical. The fairly small variability in rod settings in the upright static condition was mainly due to the subjects' slightly anticipating, or just missing, the precise moment at which the rod reached the vertical; the larger variability observed in the horizontal position reflects a genuine uncertainty, spontaneously expressed by many subjects, as to the direction of the true vertical. 
and, as in previous studies, both the control subjects and N.L. exhibited considerably greater variability in their judgments of the vertical with the head tilted into the nearhorizontal position. Although no significant increase in the mean deviation of settings from the true vertical was observed in N.L. when lying down, this may well have been due to the absence of an A effect, which in normal subjects interacts with the effect of the rotating background to produce a greater level of inaccuracy than when upright (Dichgans et al., 1974).

The major finding from this investigation is that lack of somatosensory function below the neck has a pronounced effect on the apparent tilt produced by a $90^{\circ}$ tilt of the body. The discovery that somatosensory information may play an important role in producing the A effect has implications for the mechanisms subserving perception of the upright proposed by Mittelstaedt (1983). He argued that the subjective vertical depends on two competing types of information, the first relating to the direction of gravity and the second based on the longitudinal axes of the head and body. Mittelstaedt explained the tendency to perceive the vertical as tilted in the same direction as the perceiver, referred to here as the A effect, as a personal trait or "idiotropic vector." The idiotropic vector apparently makes no contribution to active postural control, since subjects are able to actively roll themselves into a position $90^{\circ}$ from the vertical, without visual cues, with remarkable accuracy.

From this study, it would appear that the magnitude of the idiotropic vector may bear some relation to the utilization of somatosensory information in determining the direction of the apparent vertical, since the tendency to align the vertical with the true head and body axis was not manifest in N.L. This would seem to be consistent with the relatively stronger influence of the idiotropic vector under conditions in which there is a paucity of accurate visual and vestibular information-in this case, when lying sideways in a darkened room. Interestingly, the slight clockwise shift of the perceived vertical displayed by N.L. when lying down approximately matches the actual $10^{\circ}$ displacement of his head relative to his trunk. Thus, since the angle between the head and trunk is considered by Mittelstaedt to contribute to the idiotropic vector, it would appear that in the lying position, the loss of information from the trunk may even have resulted in an effective reversal in the direction of the idiotropic vector in N.L. as compared with the control subjects.

Although the evidence presented supports the idea that somatosensory information has an important influence on perception of the vertical for subjects in a static horizontal position, its precise role remains subject to debate. Howard (1982) has suggested that adaptation of somaesthetic receptors, inferred from the postural aftereffects that follow prolonged physical tilt, affects judgments of the upright. By this account, the tilt of the subjective vertical toward the body axis might result from an underestimation of the extent of body tilt due to adaptation of somatosensory receptors in the trunk. Accordingly, the subject without somatosensory function below the neck would not be expected to experience this illusion, since his judgments of orientation must necessarily be made without reference to any information from the body. Clearly, some different mechanism, presumably mediated by vestibular and/or neck receptors, would then be needed to account for the $\mathrm{E}$ effect, which dominated the settings of N.L. Moreover, it would be necessary to assume that in this study, the somaesthetic adaptation reached an asymptote within the 2 min that elapsed after the subjects had lain down and before judgments in the horizontal position were elicited, since no significant change in the magnitude of the A effect was observed as a function of trials.

This account only partially concurs with that of Mittelstaedt, who postulated that the idiotropic vector results from a complex interaction between information concerning the head and the trunk's orientation, and who maintained that perception of the visual vertical is dominated by otolithic information (Mittelstaedt \& Fricke, 1988). However, his observation that subjects can actively assume an accurate orientation relative to the vertical can easily be explained in terms of the compelling dynamic vestibular and somatosensory information provided by changes in body position, and the absence of somaesthetic adaptation during such changes. Further research is required to resolve the remaining discrepancies among current hypotheses concerning the processes mediating apparent displacement of the visual vertical in static tilted subjects.

\section{REFERENCES}

Cole, J. D., Katifi, H. A., \& Sedgwick, E. M. (1986). Observations on a man without large myelinated fibre sensory input from below the neck. Journal of Physiology, 376, 47.

Dichgans, J., Diener, H. C., \& Brandt, T. (1974). Optokineticgraviceptive interaction in different head positions. Acta Otolaryngologica, 78, 391-398.

How A RD, I. P. (1982). Human visual orientation. Chichester, England: Wiley.

MERKER, B. H., \& HELD, R. (1981). Eye torsion and the apparent horizon under head tilt and visual field rotation. Vision Research, 21, 543-547.

Miller, E. F., Fregly, A. R., \& Graybiel, A. (1968). Visual horizontal-perception in relation to otolith-function. American Journal of Psychology, 81, 488-496.

MittelstaedT, H. (1983). A new solution to the problem of the subjective vertical. Naturwissenschaften, 70, 272-281.

Mittelstaedt, H. , \& Fricke, E. (1988). The relative effect of saccular and somatosensory information on spatial perception and control. Advances in Otorhinolaryngology, 42, 24-30.

NyBORG, H. (1971). Tactile stimulation and perception of the vertical: 1. Effects of diffuse versus specific tactile stimulation. Scandinavian Journal of Psychology, 12, 1-13.

SCHONE, H. (1975). The "weight" of the gravity organ's signal in the control of perceptual and reflex type orientation at different body positions. Fortschritte Zoologie, 23, 274-285.

Stoffregen, T. A., \& Riccio, G. E. (1988). An ecological theory of orientation and the vestibular system. Psychological Review, 95, 3-14.

Young, L. R., Oman, C. M., \& Dichgans, J. M. (1975). Influence of head orientation on visually induced pitch and roll sensation. Aviation, Space \& Environmental Medicine, 46, 264-268.

(Manuscript received July 17, 1989; revision accepted for publication February $7,1990$. ) 